\title{
Validation of the Efficacy of a Practical Method for Neutrophils Isolation from Peripheral Blood
}

\author{
JONATHAN DEGEL, MASIH SHOKRANI
}

OBJECTIVE: The objectives of this study were to validate the Polymorphprep ${ }^{\mathrm{TM}}$ method for neutrophils isolation for conditions within an identified clinical laboratory setting and to confirm the purity and viability of isolated neutrophils utilizing standard clinical laboratory cell counting, differential, and viability methods.

DESIGN: A validation study, utilizing a quantitative and qualitative design, was performed.

PATIENTS /SAMPLES: 18 EDTA tube blood samples were collected from donors between the ages of 18-60, with no underlying condition. The study was performed in a CLS student laboratory of a midwestern university. The Institutional Review Board approved the study and informed consent was obtained from each subject before blood collection.

INTERVENTIONS: Blood samples were treated using a Polymorphprep ${ }^{\mathrm{TM}}$ procedure to isolate a pure population of neutrophils.

MAIN OUTCOME MEASURES: Pre and post cell counts were performed using an automated cell counter. Manual white cells differentials and Trypan blue white blood cells viability analysis was performed.

RESULTS: The mean purity levels were at least $94 \%$ following isolation with Polymorphprep ${ }^{\mathrm{TM}}$, the mean viability of neutrophils was $99 \%$.

CONCLUSION: The use of Polymorphprep ${ }^{\mathrm{TM}}$ technique provides a practical and effective method for the isolation of neutrophils from peripheral blood which can be used for investigative purposes. Validation of the purity of the isolated cell population can be readily confirmed using standard clinical laboratory cell counting and differential analysis.
ABBREVIATIONS: $\mathrm{CBC}=$ Complete blood count, EDTA = ethylenediaminetetraacetic acid, $\mathrm{WBC}=$ White blood Cells, PMN = Polymorphonuclear neutrophil, Peripheral blood mononuclear cells (PBMCs)

INDEX TERMS: Human blood, Neutrophils, Polymorphprep ${ }^{\mathrm{TM}}$, Purity, and WBC Differential.

Clin Lab Sci 2010;23(2):94

Jonathan Degel, BS, St. Louis, MO 63127

Masih Shokrani, PhD, MT(ASCP), Northern Illinois University, DeKalb, Illinois 60116-2828.

Address for Correspondence: Masih Shokrani, PhD MT(ASCP), Assistant Professor, Clinical Laboratory Sciences Program, School of Allied Health and Communicative Disorders, College of Health and Human Sciences, Northern Illinois University, DuSable Hall 165, DeKalb, Illinois60116-2828, mshokrani@niu.edu

\section{INTRODUCTION}

Human blood is made up of red blood cells (RBCs ), white blood cells (WBCs), platelets and plasma. Neutrophils make up 54-62\% of the WBCs in the peripheral blood circulation. Besides neutrophils, also called Polymorphonuclear neutrophils (PMN), there are other white blood cells in the peripheral blood. They include eosinophils (1-3\%), basophils (0-1\%), monocytes (4-10\%) and lymphocytes (20-40\%). Granulocytes are WBC that contain granules in their cytoplasm and consist of neutrophils, eosinophils and basophils in the peripheral blood. ${ }^{1}$

Neutrophils play an important role in innate immunity to protect the body and are a major contributor to inflammation. ${ }^{2,3}$ Neutrophils develop to an average size 


\section{RESEARCH AND REPORTS}

of 9-15 microns and a nucleus with 2-5 heavily clumped lobes with a thin strand connecting them. ${ }^{4}$

Isolation of neutrophils in a pure form is required in order to investigate the intricate roles that neutrophils play in infections and inflammatory disorders. ${ }^{2,3}$ However, the current methods for isolating neutrophils are time consuming and require sometimes advanced laboratory skills.

Polymorphprep $^{\mathrm{TM}}$ is a fairly novel method for the isolation of pure neutrophils from whole blood. Polymorphprep ${ }^{\mathrm{TM}}$ is a sterile and endotoxin tested solution. The density gradient medium, Polymorphprep ${ }^{\mathrm{TM}}$, is customized to the simultaneous purification of neutronphils, PMNs, and peripheral blood mononuclear cells (PBMCs) ( manufactured by AXIS- SHIELD, Oslo, Norway and distributed by Greiner Bio- One, Monroe, North Carolina).

The separation procedure uses an isoosmotic density barrier that separates the mononuclear cells and neutrophils into two distinct bands. As Figure 1 illustrates, the first band contains the mononuclear cells below the plasma. A small layer of Polymorphprep ${ }^{\mathrm{TM}}$ is below the mononuclear cells, followed by the band of neutrophils, and then followed by more Polymorphprep $^{\mathrm{TM}}$. The RBC settles to the bottom of the tube due to the density barrier created by the solution. ${ }^{5,6}$

The objectives of this study were to validate the Polymorphprep $^{\mathrm{TM}}$ method for neutrophils isolation for conditions within an identified clinical laboratory setting and to confirm the purity and viability of isolated neutrophils utilizing standard clinical laboratory cell counting, differential, and viability methods.

\section{MATERIALS AND METHODS}

1. Polymorphprep ${ }^{\mathrm{TM}}$ is a ready-made, sterile and endotoxin tested solution for the isolation of pure PMNs from whole blood. Polymorphprep ${ }^{\mathrm{TM}}$ solution contains $13.8 \%(\mathrm{w} / \mathrm{v})$ sodium diatrizoate and $8.0 \%(\mathrm{w} / \mathrm{v})$ polysaccharide. Polymorphprep ${ }^{\mathrm{TM}}$ is produced by AXIS-SHIELD, Oslo, Norway and distributed by Greiner Bio-One, Monroe, North Carolina

2. $5 \mathrm{~mL}$ EDTA blood collection tubes

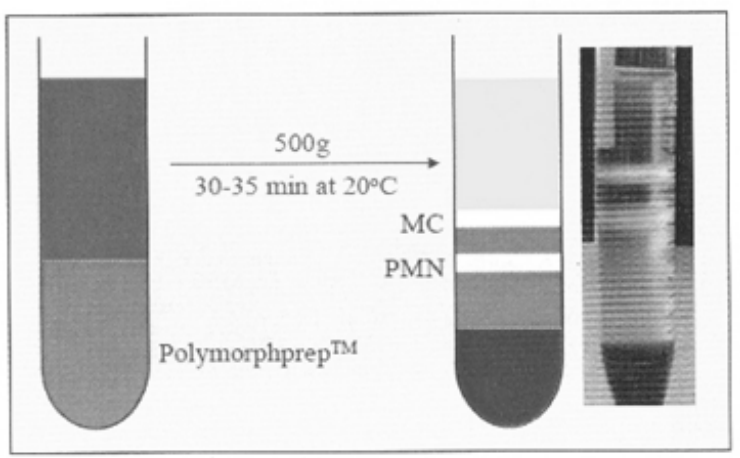

Figure 1. Purification of PMNs using Polymorphprep ${ }^{\mathrm{TM}}, \mathrm{MC}$ (mononuclear cells), PMNs polymorophonuclear leukocytes ( reproduced with permission from AXIS-SHIELD, Oslo, Norway).

3. Abbott CELL-DYN 1700, a 3-part leukocytes automated differential cell counter (Abbott Diagnostics, Abbott Park, Illinois)

4. Phillips Drucker L-708 Combination Centrifuge

5. Peripheral blood smears were prepared by Cytospin (Shandon Cytospin-2 TM Waltham, Massachusetts) and stained with Wrights Stain for manual differential counts of WBC.

6. Hemacytometer

7. Polymorphprep ${ }^{\mathrm{TM}} \&$ Identification of neutrophils: Human Peripheral blood was collected in $5 \mathrm{~mL}$ EDTA tubes from healthy donors. A complete blood count (CBC) was performed on an automated cell counter CELL-DYN 1700. The parameters used for this investigation were the total WBC and granulocyte counts, as counted by a 3part differential automated cell counter. A pipette was used to place $5 \mathrm{ml}$ of the Polymorphprep ${ }^{\mathrm{TM}}$ solution into a $15 \mathrm{~mL}$ conical tube. Five $\mathrm{mL}$ of blood from the donor was layered on top of the Polymorphprep ${ }^{\mathrm{TM}}$ and centrifuged at $500 \mathrm{~g}$ for 40 minutes at room temperature in order to achieve a maximum displacement of the RBC to the bottom of the tube. A sterile pipette was used to remove plasma and mononuclear cells from the upper band. Neutrophils were aspirated with a $1 \mathrm{~mL}$ pipette and placed in a $10 \mathrm{~mL}$ tube. Eight $\mathrm{mL}$ of saline solution was added to the extracted cells and the remaining Polymorphprep ${ }^{\mathrm{TM}}$ solution was centrifuged for 10 minutes at $400 \mathrm{~g}$. Next, saline was removed and the pelleted cells were resuspended in $1 \mathrm{~mL}$ of saline. $100 \mu \mathrm{l}$ of the recovered 


\section{RESEARCH AND REPORTS}

cell suspension were used to perform a Cytospin for 10 minutes at 600 revolutions per minute. Cytospin method uses a low centrifugal force to separate and deposit a monolayer of cells on slides while maintaining cellular integrity. The slides made from the Cytospin were Wright stained and a manual differential count was performed on each slide using $100 \mathrm{x}$ oil immersion (for a total magnification of 1000 fold). In addition, viability of isolated neutrophils was tested with Trypan blue solution and percentage of viable cells was counted using a hemocytometer at a 400 fold total magnification.

8. The mean and standard deviation for the CBC and differential counts were calculated. Neutrophils make up for over $90 \%$ of the granulocytes counted by the CELL-DYN 1700. The number of granulocytes recovered from each blood sample was calculated before and after isolation by the CELLDYN 1700.

The purity was examined by the number of granulocytes in comparison with the number of remaining cells and by a microscopic differential of isolated cells. The percentage of granulocytes that were recovered was calculated. The calculation was determined by dividing the final number of granulocytes multiplied by the dilution factor, dilution factor was 1 since $1 \mathrm{~mL}$ of saline was added to neutrophils, divided by the original granulocytes numbers multiplied by 5 , which was the original volume of blood used. In addition, a mean for viability of cells was determined to be about $99 \%$.

\section{RESULTS}

Table 1 shows the percent of neutrophils by a manual differential on the post treatment sample, and the initial and final granulocyte counts by the CELL DYN 1700.

Results for isolating neutrophils using Polymorphprep ${ }^{\mathrm{TM}}$ and a 40-minute centrifugation time showed mean purity levels of at least $94 \%$ with a standard deviation of 4.91 (Table 1). Cell viability was determined by Trypan blue exclusion test using a hemacytometer. Unlike dead cells that pick up the dye, live cells have intact cell membrane that excludes dyes such as Trypan blue. Cells were counted for viability using Trypan blue exclusion test in a hemacytometer.
After every isolation, viable (unstained) cells were determined to be about $99 \%$ of total cells.

Figure 2 illustrates the Cell DYN 1700 WBC histograms before and after isolation with Polymorphprep $^{\mathrm{TM}}$ for a representative sample (Figure $2 \mathrm{~A}$ and Figure $2 \mathrm{~B}$, respectively). The peaks are representative of the different concentrations of WBC. The peaks follow in the order of lymphocytes, monocytes and granulocytes. Figure 2B displays how one cell population is isolated by the peak in the granulocyte region. Results for the WBC histograms after isolation showed a large broad peak in the granulocyte regions resulting in high levels of purity, while smaller less broad peaks contained a lower percentage of granulocytes and resulted in lower levels of purity.

Figure 3 demonstrates the pure population of neutrophils obtained at the post isolation with the Polymorphprep $^{\mathrm{TM}}$ procedure. Figure 3 shows 2 representative samples on a Cytospin prepared and Wright stained for manual differential (for a total magnification of 600 fold).

\section{DISCUSSION}

The Polymorphprep ${ }^{\mathrm{TM}}$ procedure went through many test runs in order to optimize conditions. Different methods for obtaining the band of neutrophils (PMNs) from the conical tube were attempted with mixed results. The best method of retrieval was to aspirate some of the layers above and below the PMNs to obtain as many PMNs as possible. Three aspirations were used on average to isolate the cells. The amount of centrifugation time for the tubes before removal of the layers was increased to 40 minutes, when it became clear that the amount of RBCs that would settle to the bottom of the tube would increase and would allow for an easier separation. The purity levels obtained using a 40-minute centrifugation averaged at least 94\%. The level of purity for a 40-minute centrifugation time would have been even higher, had it not been for two or three outliers from irregular staining of cells prior to manual differential. The levels of purity achieved com pared favorably to previous experiments using Polymorphprep $^{\mathrm{TM}}$ ? 
RESEARCH AND REPORTS

Table 1. Granuloctyes counts by CELL-DYN 1700. Treatment with Polymorphprep ${ }^{\mathrm{TM}}$ (40-Minute spin time, $\mathrm{n}=18$ )

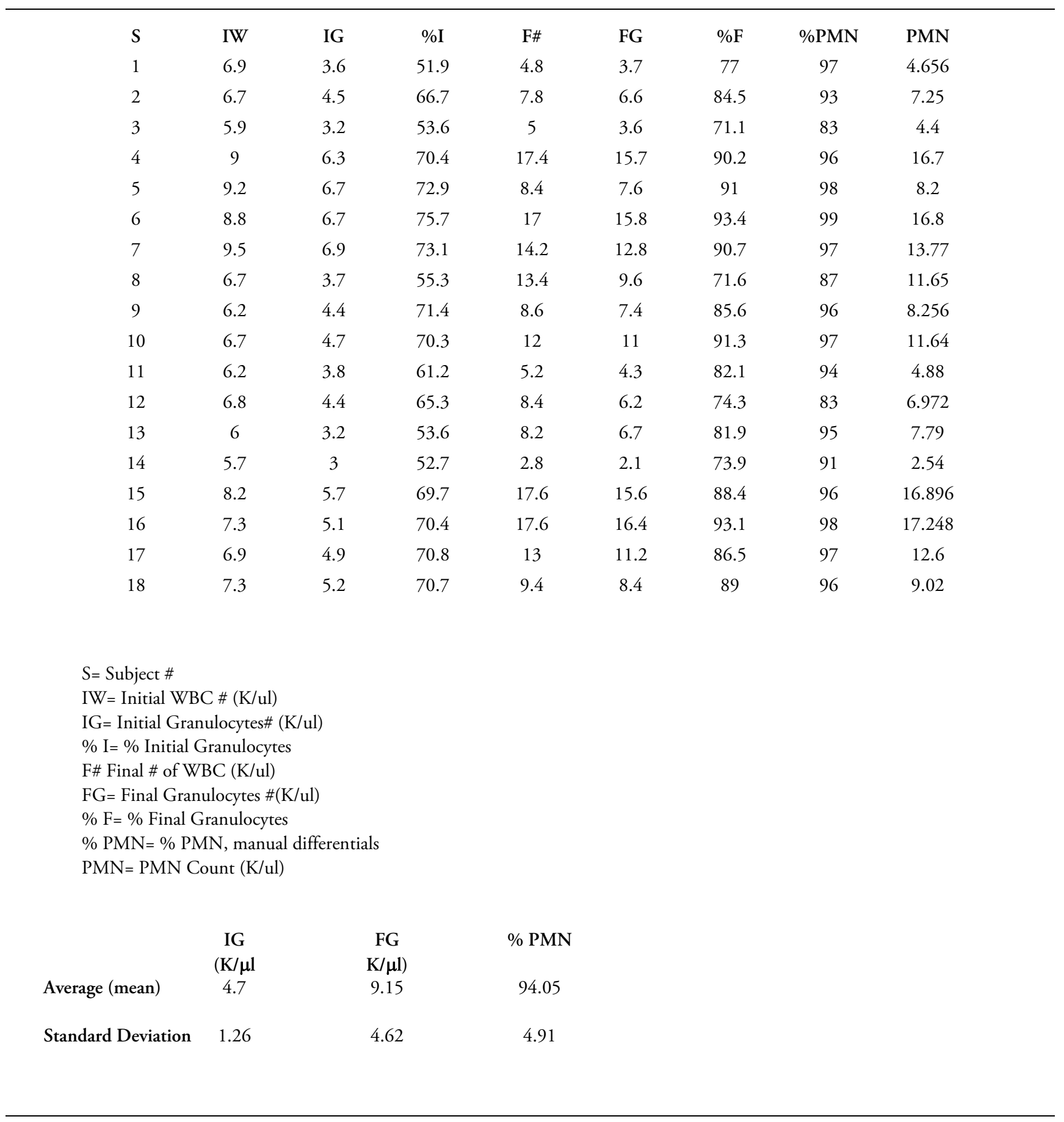




\section{RESEARCH AND REPORTS}

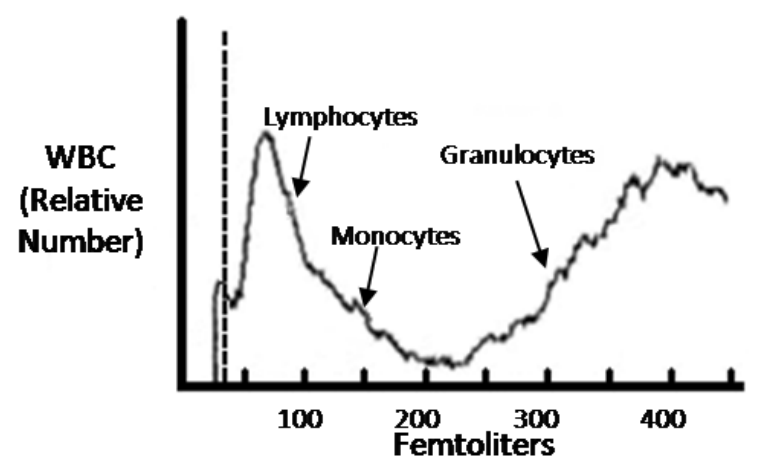

Figure 2A. WBC Histogram on CELL 1700 before isolation of PMNs

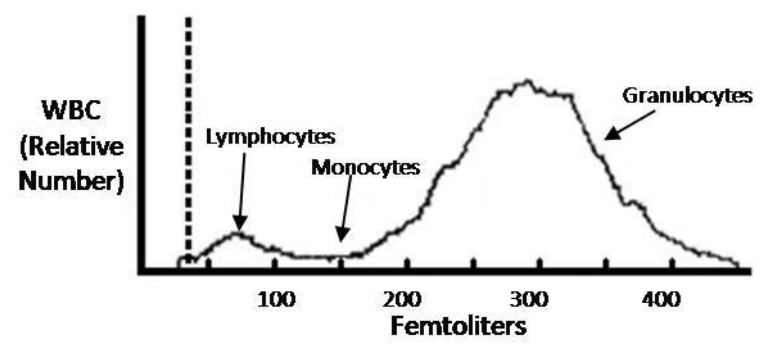

Figure 2B. WBC Histogram on CELL 1700 after isolation of PMNs

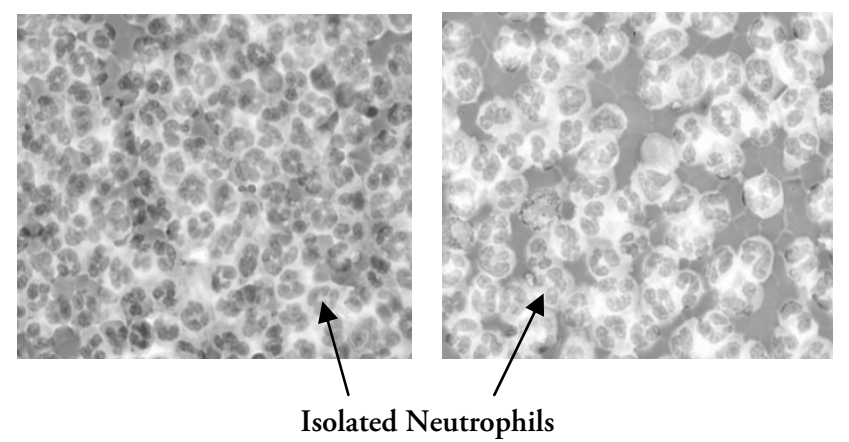

Figure 3. Neurtophils in a post Polymorphprep ${ }^{\text {TM }}$ Isolation (2 representative samples Cytospin prepared and Wright stained)

This study was performed with minimum supplies and laboratory space available. A 3-part automated cell counter measures WBC histogram and counts for lymphocytes, monocytes, and granulocytes. The granulocyte region of a histogram represents neutrophils, eosinophils, and basophils. Neutrophils comprise more than 90 percent of granulocytes. A 5-part automated cell counter differentiate among granulocytes and reports neutrophils, eosinophils and basophils in addition to lymphocytes and monocytes. ${ }^{1}$ For experimental purposes, determination of granulocytes is a measure of neutrophil counts, as measured by a 3-part automated cell counter. The cell counter used for this study was a CELL-DYN 1700 which is a 3-part leukocytes differential analyzer. The use of a 5-part leukocyte differential analyzer would have been more beneficial for the verification of purity of neutrophils. The Polymorphprep ${ }^{\mathrm{TM}}$ method accomplished the goal of this study to obtain a high purity of viable neutrophils. Future studies will focus on functional studies, such as test for chemotaxis, for isolated neutrophils.

\section{CONCLUSION}

The Polymorphprep ${ }^{\mathrm{TM}}$ proved to be a practical method with high efficacy. The results of this study indicate isolation of highly pure neutrophils from peripheral blood averaging at least $94 \%$ and a viability level greater than $99 \%$. Use of more advanced centrifuge for neutrophils isolation can even increase the purity to much higher levels.

This work introduces practical ways for the isolation and assessment of a pure population of neutrophils from peripheral blood for investigative purposes using materials and methods available in a standard clinical laboratory setting.

\section{REFERENCES}

1. McKenzie, Shirlyn B. Clinical Laboratory Hematology. Upper Saddle River, New Jersey: Pearson Education; 2004

2. Christopher MJ. Link DC. Regulation of neutrophil homeostasis. Curr. Opin. Hematol. 2007; 14(1): 3-8.

3. Barnes PJ. New molecular targets for the treatment of neutrophilic diseases. J. Allergy. Clin. Immunol 2007; 119(5): 1055-62.

4. HoffBrand A. V, Moss P.A.H, Petit J.E. Essential Haematology. Massachusetts: Blackwell Publishing; 2006

5. Boyum, A. (1968) Scand. J. Clin. Lab. Invest. 1968; 21, Suppl 9

6. Ferrante A, Thong, Y.H. Optimal conditions for simultaneous purification of mononuclear and polymorphonuclear leucocytes from human blood by the Hypaque- Ficoll methods. J. Immunol. Meth 1980; 36: 109-117

7. Iwabuchi K, Nagaoka I. Lactosylceramide- enriched glycosphingolipid signaling domain mediates superoxide generation from human neutrophils. Blood. 2002; 100(40): 1454-6 